\title{
Quantitative characteristics of traps in AIGaN/GaN MIS-HEMT via transient capacitance measurement
}

\author{
Jie Lin ${ }^{1,2}$, Bin Dong ${ }^{1,2}$, Ning Wang ${ }^{1,2}$, Zongdai Liu ${ }^{1,2}$, Lingli Jiang ${ }^{1,2}$, Kai Cheng ${ }^{2}$ and Hongyu Yu $u^{1,2, a}$ \\ ${ }^{1}$ Department of Electrical and Electronic Engineering, South University of Science and Technology of China, \\ Shenzhen, 518055, China \\ ${ }^{2}$ Shenzhen Key Laboratory of 3rd Generation Semiconductor Devices, Shenzhen, China \\ ${ }^{3}$ Enkris Semiconductor Inc., Suzhou, 215123, China
}

\begin{abstract}
The electrical property degradation of AlGaN/GaN MIS-HEMT, including degradation of output characteristics, a higher ON-resistance and a threshold voltage negative shift $(\sim 1.8 \mathrm{~V})$, is correlated with near-interface trap behaviours in SiN/AlGaN. These traps are quantitatively characterized using the transient capacitance measurement, from which we could estimate the near-interface trap densities in the SiN/AlGaN. Measurements reveal the trap density before step-stress with $1.404 \times 10^{15} \mathrm{~cm}^{-3}$ and an increased density of $1.709 \times 10^{15} \mathrm{~cm}^{-3}$ after step-stress. A hot electron injection model is used to discuss the relationship between trap behaviours and device reliability.
\end{abstract}

Keywords: AlGaN/GaN MIS-HEMT; transient capacitance measurement; traps.

\section{Introduction}

AlGaN/GaN-based high electron mobility transistor (HEMT) is of great interest because of its outstanding performance under high power, high frequency and high-temperature conditions, which enables applications in the field of power grid, automobiles, wireless communication, etc. However, the reliability of device is still a problem that people take great concern [1]. The reliability problems, including current collapse [2], threshold voltage shift [3] and an increase of the gate/drain leakage current [4], are dominated primarily by the traps inside the device [5]. Thus, it is necessary to explore the relationship between trap behaviors and device reliability. Although many research groups have demonstrated several excellent methods for studying device degradation and reliability in AlGaN/GaN HEMTs such as low-frequency noise measurements [6-9], stress testing [10-11] and deep-level optical spectroscopy (DLOS) [12], few of them have quantitatively investigated the relationship between trap density and device reliability. In this work, we demonstrate the capability to quantitative analysis the change of trap density before and after applied electrical step-stress, a possible explanation of the trapping mechanism in the device is also proposed.

\footnotetext{
${ }^{\mathrm{a}}$ Corresponding: yuhy@sustc.edu.cn
} 


\section{Experiments}

The AlGaN/GaN MIS-HEMTs have been grown on a 6-inch GaN-on-Si substrate by metal organic chemical vapor deposition (MOCVD). The barrier layer is 22nm with the composition of $22 \% \mathrm{Al}$. The structure of the device is shown in Figure 1 (a). The multi-fingered gate width $8.5 \mathrm{~mm}$ of the periphery MIS-HEMT and the gate length is $2 \mu \mathrm{m}$, the gate dielectric thickness is $35 \mathrm{~nm}$. The gate-drain distance $\left(L_{G D}\right)$ is $10 \mu \mathrm{m}$ and the gate-source $\left(L_{G S}\right)$ is $2 \mu \mathrm{m}$.

(a)
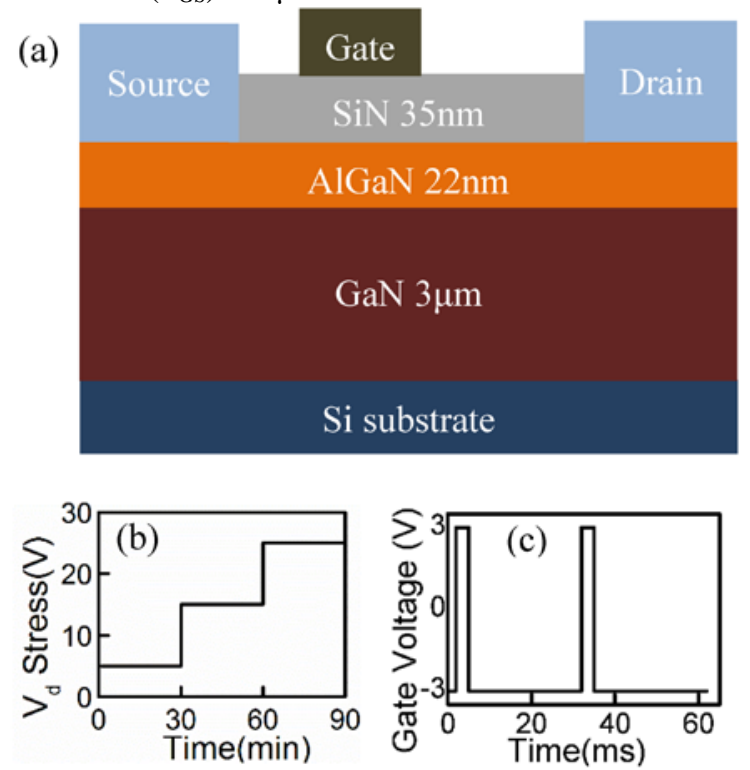

Figure 1. (a) Schematic structure of AlGaN/GaN MIS-HEMT. (b) The solid line indicates the step-stress from $5 \mathrm{~V}$ to $25 \mathrm{~V}$ applied to the drain terminal while the gate is $-15 \mathrm{~V}$ biased, device is OFF-state. (c) Pulse cycle applied to the gate for transient capacitance measurement.

Applied electrical stress to the device could induce both temporary and permanent current collapse because of electron trapping in $\mathrm{AlGaN} / \mathrm{GaN}$ HEMTs [13]. In this study, a negative bias of $-15 \mathrm{~V}\left(V_{G}\right)$ was applied to the gate electrode, and $V_{D}$ increased from $5 \mathrm{~V}$ to $25 \mathrm{~V}$ with a step of $5 \mathrm{~V}$ to study the effect of electric stress induced trap in AlGaN/GaN MIS-HEMT. The test was performed in the dark at room temperature to avoid the stress recovery of device induced by illumination. Stressing was stopping at each 30-minute and DC characterizations were measured. A step-stress protocol is shown in Figure 1 (b).

Transient capacitance method was used to study the trapping behaviours. Source and drain terminals were grounded while a pulse (Figure 1 (c)) was applied to the gate before the transient capacitance measurement. When the pulse voltage was up to $3 \mathrm{~V}$, a corresponding increase was in the depletion capacitance. The traps were filled with electrons from the conduction band. The width of depletion region increased with reverse pulse voltage to $-3 \mathrm{~V}$, so the capacitance decreases. As electrons were emitted from trap states, the width of depletion region became slightly smaller and the junction capacitance went back to the previous value of steady state. The traps filling and the emptying process could occur with a junction capacitance changing over time.

\section{Results and discussions}

Output $I$ - $V$ characteristics before and after step-stressed on the drain were shown in Figure 2 (a).Onstate drain and off-state drain leakage current increased after applying electrical stressing. When the gate voltage was back to 0 , the slope of $I_{D}-V_{D}$ was measured to obtain on-state resistance after each stress step, the ratios of ON-state resistance changes at each stress step were presented in Figure 2 (b). 
Figure 3. (a) shows the transfer characteristics of the device before and after applied step stress, respectively. It can be seen in Figure 3 (b) that the threshold voltage shifting from $-7.7 \mathrm{~V}$ to $-9 \mathrm{~V}$ after stressed
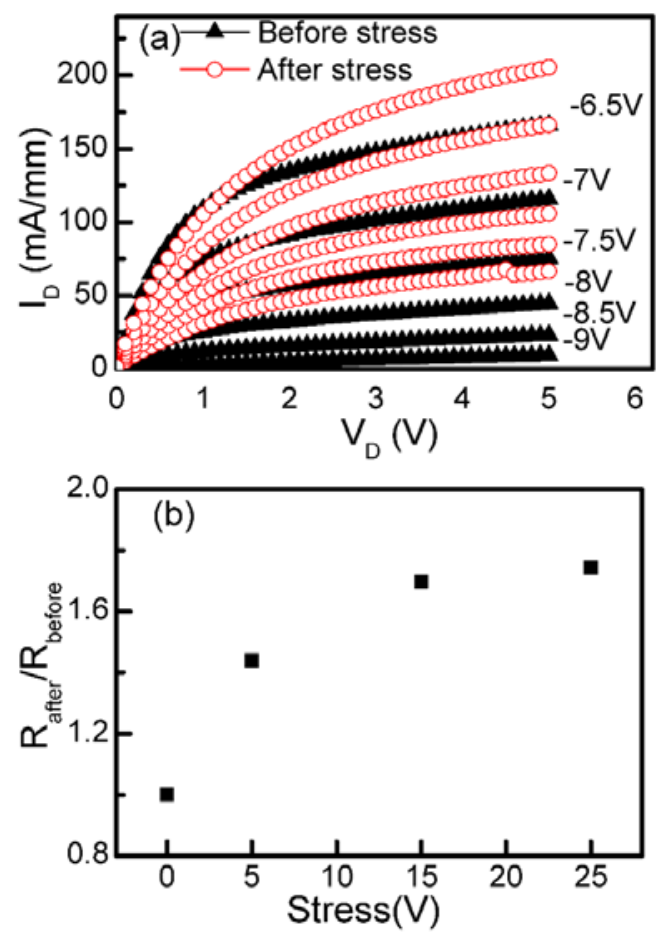

Figure 2. (a) $I_{d}-V_{d}$ characteristics performed on AlGaN/GaN HEMTs before and after stressed of Vgs from -6.5V to $-9 \mathrm{~V}$. (b) ON-state resistance increases after every step-stress

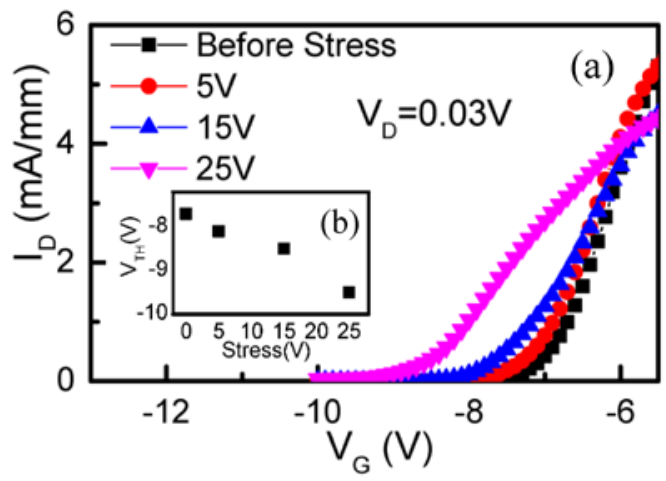

Figure 3. (a) Transfer characteristics of AlGaN/GaN HEMTs after each step-stress. (b) Threshold voltage shifts negative with the stress increasing.

The Capacitance-Voltage $(C-V)$ characteristics of AlGaN/GaN HEMTs were measured on AlGaN/GaN HEMTs before stressed at room temperature using a capacitance meter of $1 \mathrm{MHz}$ frequency. Doping concentration $N_{D}$ is related to $C$ and $V$ :

$$
1 / C^{2}=2 V /\left(A^{2} \varepsilon e N_{D}\right)
$$

Where $A$ is the surface of the Schottky contact, $\varepsilon$ is the dielectric constant and $e$ is the elementary charge. Formula (1) indicates that, $1 / C^{2}$ is a linear function of gate voltage $V$ near the value of 
threshold voltage $(\sim-8 \mathrm{~V})$, as shown in Figure 4 . The net doping concentration $N_{D}=9.081 \times 10^{15} \mathrm{~cm}^{-3}$ was determined from the relation of $1 / C^{2}$ and $V$.

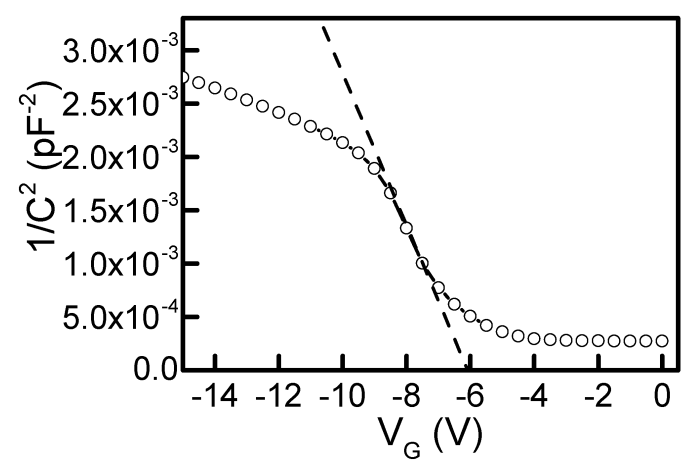

Figure 4. The relation of $1 / C^{2}$ and $V$ obtained on AlGaN/GaN HEMT before stressed at room temperature.

The capacitance transient characteristics before and after step-stressed are shown in Figure 5. The transient change of capacitance $\Delta C$ was calculated from a time window of $15 \mathrm{~ms}$. Before step-stressed, the value of $\Delta C$ was $9.2 \mathrm{pF}$. After step-stressed, $\Delta C$ increased to $11.2 \mathrm{pF}$ due to the step-stress induced extra traps. The trap density was estimated based on the formula [14]:

$$
\Delta C / C_{0}=N_{T} / 2 N_{D}
$$

Where $C_{0}$ is the capacitance value of steady state. The calculated trap density before and after electrical stressing are $1.404 \times 10^{15} \mathrm{~cm}^{-3}$ and $1.709 \times 10^{15} \mathrm{~cm}^{-3}$ respectively. The results are summarized in Table 1.

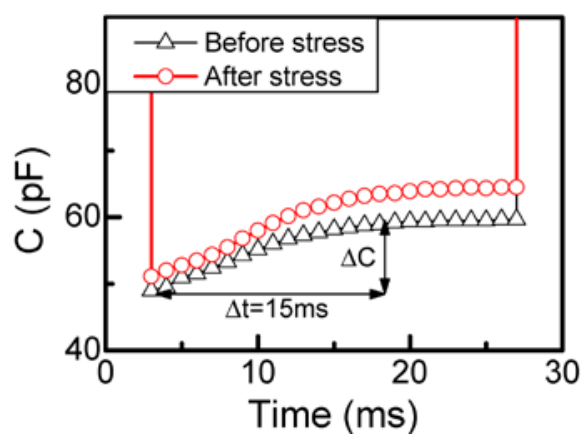

Figure 5. Capacitance transient characteristics of AlGaN/GaN HEMT after 25V stressed

Table 1 . The change in transient capacitance and the corresponding trap density

\begin{tabular}{|c|c|c|}
\hline & $\Delta \mathrm{C}$ & Trap density \\
\hline Before stressed & $9.2 \mathrm{pF}$ & $1.404 \times 10^{15} \mathrm{~cm}^{-3}$ \\
\hline After stressed & $11.2 \mathrm{pF}$ & $1.709 \times 10^{15} \mathrm{~cm}^{-3}$ \\
\hline
\end{tabular}

After applied electrical stressing, a significant degrading of conductive ability and threshold voltage shift negatively were observed. The transient capacitance measurement connected with an increment of deep level defects concentration $\left(\Delta N_{T} \sim 0.305 \times 10^{15} \mathrm{~cm}^{-3}\right)$. These results indicate that the applied stress bias on drain could cause the accumulation of the deep level traps. The drop of drain current was partly related to the increase of ON-state resistance, and the increase of drain leakage was due to the threshold voltage negatively shifting, which weakens the ability of gate to control drain- 
source current because the gate needs a higher negative bias to turn off the device. The shift of threshold voltage after stressed indicates that the interface state under gate was influenced by electrical stressing. Deep level traps were induced in the near-interface of SiN/AlGaN during electrical stressing, which causes a degradation of ability to gate control and a change in sheet carrier concentration in the channel. Generally, this mechanism is combined with a shift positively of threshold voltage $[15,16]$, but in our experiment, threshold shift negatively under stressed. Hot electron degradation is a reliability failure mechanism [17]. When devices are biased at high $V_{D S}$, due to the high electric field present in the gate-drain region, electrons gain sufficient kinetic energy to overcome a potential barrier necessary to break an interface state, significant hot electron phenomena happen. According to hot electron injection model, the accumulation of traps stimulates gate leakage increasing. Otherwise, high density of defects lowers the carrier mobility and increase the ONresistance. Thus, the conductive ability declines. New generated traps due to the stressed in the AlGaN barrier near the interface release electrons, resulting in the threshold voltage negative shift and an increase in drain resistance. With the increment of applied bias, the hot electron is enhanced, which leads to a higher gate leakage. Thus, the threshold voltage continuously shifts negatively and then causes the degradation of AlGaN/GaN MIS-HEMT device after step stressed.

\section{Conclusion}

In summary, traps were located at near-interface of SiN/AlGaN layer and characterized by using a transient capacitance method. The change of trap density before and after step-stressed was quantitatively analyzed. After stressed, ON-resistance increased by 1.7 times, a significant fall of conductive ability and threshold voltage $(\sim 1.8 \mathrm{~V})$ negative shift were observed. Transient capacitance measurement reflected an increment of deep level defects concentration $\Delta N_{T}$ with $0.305 \times 10^{15} \mathrm{~cm}^{-3}$. These results indicate that: a) the stress bias on drain could result in the accumulation of deep level traps. According to hot electron model, the accumulation of traps stimulated gate leakage increasing. Otherwise, high density of defects lowered the carrier mobility and increased the device resistance. Thus, the conductive ability declined. b) New generated traps in the near-interface of SiN/AlGaN layer released electrons, resulting in the negative shift of threshold voltage. With the increment of applied bias, the hot electron injection was enhanced, which led to a large gate leakage. Thus, the threshold voltage continuously shifted negatively.

\section{Acknowledgement}

The authors acknowledge the funding support of Key laboratory of third-generation semiconductor devices in Shenzhen (Project No. ZDSYS20140509142721434), 2014-084 Key Technology Development of Si based GaN power devices (Project No.JSGG20140729145956266) and Energyefficient Si based GaN power devices (Project No. KQCX20140522151322946).

\section{Reference}

1. N. Killat, M. Montes Bajo, T. Paskova, K. R. Evans, J. Leach, X. Li, Ü. Özgür, H. Morkoç, K. D. Chabak, A. Crespo, J. K. Gillespie, R. Fitch, M. Kossler, D. E. Walker, M. Trejo, G. D. Via, J. D. Blevins and M. Kuball, Appl. Phys. Lett. 103, 193507 (2013).

2. Takashi Katsuno, Takaaki Manaka, Tsuyoshi Ishikawa, Hiroyuki Ueda, Tsutomu Uesugi and Mitsumasa Iwamoto, Appl. Phys. Lett. 104, 252112 (2014).

3. Tian-Li Wu, Denis Marcon, Benoit Bakeroot, Brice De Jaeger, H. C. Lin, Jacopo Franco, Steve Stoffels, Marleen Van Hove, Robin Roelofs, Guido Groeseneken and Stefaan Decoutere, Appl. Phys. Lett. 107, 093507 (2015).

4. YongHe Chen, XiaoHua Ma, WeiWei Chen, Bin Hou, JinCheng Zhang and Yue Hao, AIP Advances 5, 097154 (2015). 
5. Jie Yang, Sharon Cui, T. P. Ma, Ting-Hsiang Hung, Digbijoy Nath, Sriram Krishnamoorthy and Siddharth Rajan, Appl. Phys. Lett. 103, 173520 (2013).

6. Hemant Rao and Gijs Bosman, J. Appl. Phys. 106, 103712 (2009).

7. Cemil Kayis, C. Y. Zhu, Mo Wu, X. Li, Ümit Özgür and Hadis Morkoç, J. Appl. Phys. 109, 084522 (2011).

8. T. Roy, E. X. Zhang, Y. S. Puzyrev, X. Shen, D. M. Fleetwood, R. D. Schrimpf, G. Koblmueller, R. Chu, C. Poblenz, N. Fichtenbaum, C. S. Suh, U. K. Mishra, J. S. Speck and S. T. Pantelides, Appl. Phys. Lett. 99, 203501 (2011).

9. Sahoo D, Lal R, Kim Hyungtak, Tilak V, Eastman L, Electron Devices, IEEE Transactions on, 50(5): 1163-1170 (2003).

10. M. Tapajna, O. Hilt, E. Bahat-Treidel, J. Würfl and J. Kuzmík, Appl. Phys. Lett. 107, 193506 (2015).

11. Byung-Jae Kim, Ya-Hsi Hwang, Shihyun Ahn, Weidi Zhu, Chen Dong, Liu Lu, Fan Ren, M. R. Holzworth, Kevin S. Jones, Stephen J. Pearton, David J. Smith, Jihyun Kim and Ming-Lan Zhang, Appl. Phys. Lett. 106, 153504 (2015).

12. Nakano Y, Irokawa Y, Takeguchi M, Applied physics express, 1(9): 091101 (2008).

13. M. J. Anand, G. I. Ng, S. Arulkumaran, C. M. Manoj Kumar, K. Ranjan, S. Vicknesh, S. C. Foo, B. Syamal and X. Zhou, Appl. Phys. Lett. 106, 083508 (2015).

14. M. Charfeddine, Malek Gassoumi, H. Mosbahi, C. Gaquiére, M. A. Zaidi, H. Maaref, J. Mod. Phys. 2, 10 (2011).

15. Hemant Rao and Gijs Bosman, J. Appl. Phys. 108, 053707 (2010).

16. Zeng, Chang, Yuansheng Wang, Xueyang Liao, Ruguan Li, Yiqiang Chen, Ping Lai, Yun Huang, Yunfei En, in Reliability, Maintainability and Safety (ICRMS), 2014 International Conference on. Guangzhou, China (August 6 -8, 2014), pp. 298 - 301.

17. Gaudenzio Meneghesso, Alvise Mion, Youcef Haddab, Maura Pavesi, Manfredo Manfredi, Claudio Canali and Enrico Zanoni, J. Appl. Phys. 82, 5547 (1997). 